\title{
Estratigrafía y componentes de un fardo funerario de la cultura Chancay procedente de Cerro Colorado, Huacho
}

\author{
Pieter van Dalen Luna \\ Universidad Nacional Mayor de San Marcos \\ pvandalen2@hotmail.com \\ Łukasz Majchrzak \\ Instituto de Arqueología, Jagiellonian University. \\ archeoluk@gmail.com
}

\section{RESUMEN}

Durante las excavaciones realizadas en el sitio de Cerro Colorado (costa central peruana), Pieter van Dalen descubrió que alrededor del $40 \%$ de los 1500 enterramientos contenían restos humanos envueltos en fardos funerarios. El estudio del material asociado, junto con la datación por radiocarbono, reveló que el cementerio fue utilizado por la sociedad conocida hoy en día como la cultura Chancay (ca. 1000 a 1572 d. C.). En este artículo presentamos la estratigrafía de uno de los fardos funerarios excavados. Su diseńo informa acerca de la complejidad del ritual funerario durante el cual se realizó, así como el patrón funerario general de los Chancay.

Palabras clave: arqueología de la muerte, patrón funerario, cultura Chancay, valle de Huaura, fardos, estratigrafía.

\section{Stratigraphy and components of a funeral fardo of culture Chancay from Cerro Colorado, Huacho}

\begin{abstract}
During the excavations conducted on the Cerro Colorado site (Peruvian Central Coast), Pieter van Dalen discovered that some $40 \%$ of 1500 burials contained human remains wrapped in funerary bundles (fardos). The study of the associated material, along with radiocarbon dating, revealed that the cemetery was used by the society known today as the Chancay culture (ca. 1000 to $1572 \mathrm{AD}$ ). In this article we present the stratigraphy of one of the excavated funerary bundles. Its layout informs about the complexity of the funerary ritual during which it was made, as well as the general Chancay mortuary pattern.
\end{abstract}

KEYworDs: archeology of death, funerary pattern, Chancay culture, Huaura valley, bales, stratigraphy. 


\section{Introducción}

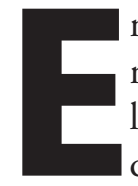
ntre los años 2014 y 2017, Pieter van Dalen realizó excavaciones en el complejo arqueológico de Cerro Colorado, en la margen izquierda del valle bajo del río Huaura (Costa Norcentral Peruana). Como resultado de estas investigaciones recuperó más de 1500 contextos funerarios de la cultura Chancay, de los cuales cerca del $40 \%$ contenía restos humanos envueltos en fardos funerarios hechos de textiles y materiales vegetales. Aunque el análisis del material aún está en proceso, ya hemos obtenido algunos datos importantes sobre el diseño de los fardos, y uno de ellos se presenta a continuación.

El cementerio perteneció a la cultura Chancay (ca. 1000 a 1572 d. C.), y funcionó probablemente desde mediados del Horizonte Medio (aprox. 700 a 1000 d. C.), durante la mayor parte del Período Intermedio Tardío (ca. 1000 a 1470 d. C.), siendo ocupado hasta el Horizonte Tardío, el período en que el imperio inca conquistó y gobernó esa área (ca. 1470 a 1533 d. C). Los estudios realizados por Krzanowski (1991, 2008) y van Dalen (2008, 2012, 2013, 2016a, 2016b, 2017) muestran que las personas de Chancay levantaron estructuras públicas y religiosas monumentales que aún son visibles en sitios enormes como Pisquillo Chico en el valle de Chancay. También se lograron tejedores, quizás incluso los mejores en toda la región central de los Andes (Bastiand 2000, Rowe 2014). Desafortunadamente, los primeros documentos en español sobre esa región revelan pocos datos (Rostworowski 2002: 269-373; van Dalen 2016a), y casi todos los cementerios de Chancay han sido fuertemente saqueados. Por esa razón, los contextos excavados por van Dalen, estudiados ahora con la aplicación de métodos químicos y biológicos modernos, pueden contribuir al conocimiento general de las costumbres prehispánicas de los pueblos andinos.

\section{La tradición de los fardos funerarios en los Andes centrales}

La tradición de los fardos funerarios en los Andes Centrales, la encontramos como una larga tradición presente en los entierros en la cultura Paracas (aprox. 800 a 200 d. C.) en la Costa Sur del Perú y se exten- dió en adelante por todos los Andes Centrales (Tello 2009). Esto ya había sido advertido por los cronistas coloniales tempranos (Cieza 1946 [1553]: 351-353, Poma 1962 [1615]: 206-211; Cobo 1964 [1635]: 163-165). Bartolomé de las Casas, elaboró una descripción de la fabricación de fardos. Según este autor, el ritual era público y tenía lugar en un patio, en cuyo centro se colocaba el cadáver. Durante la ceremonia, un narrador relataba los hechos del fallecido, acompañado por melodías tristes de las flautas. Grupos de dolientes, algunos de ellos con objetos en las manos, se acercaban al cadáver por turnos, caminando alrededor de él continuamente, colocando textiles en el cuerpo, llorando y cantando. Se hacían ofrendas votivas de alimentos, así como otros objetos utilizados por el personaje en vida. Estas ofrendas eran renovadas cada cierto tiempo al interior de la tumba (van Dalen 2017). Algunas presas de carne horneada se colgaban delante del cuerpo, y los sacerdotes comprobaban la forma en que cambiaba el color de la carne, lo que auguraba el destino de los muertos en el más allá. El luto podía durar de cinco a diez días, dependiendo del rango de fallecidos en la vida, luego el fardo terminado era enterrado en la tumba subterránea. Para facilitar más ofrendas después del funeral, los dolientes a veces levantaban construcciones sobre la tumba, en el suelo (de las Casas 1892 [1550]: 118-124). El autor nunca había estado en los Andes Centrales, y esta descripción probablemente le fue entregada por su amigo, Fray Domingo de Santo Tomás, quien pasó más de 30 años en el Virreinato del Perú, especialmente en los pueblos del valle de Chancay, donde fue cura doctrinero (antiguo territorio de la cultura Chancay). Pablo Josef de Arriaga (1968 [1621]), el famoso extirpador de la idolatría, testificó que los cuerpos de los antepasados, llamados "mallqui», eran venerados por sus descendientes y parientes como seres divinos. Este culto a los antepasados incluía la interacción física con los muertos, cambiarse de ropa, limpiar el cuerpo y hacer ofrendas de comida. En el período Colonial Temprano, el término "mallqui» significaba "una planta joven para poner en un suelo» $\mathrm{o}$ "todo árbol frutal», y en general se refería a la actividad de siembra y semillas (Holquin 2007 [1608]: 41).

Según los arqueólogos, la fabricación de bultos se percibía como la transformación de los muertos en un ancestro poderoso que poseía poder generativo (Fung 1960, Kaulicke 2001, Morales 1998, 
Makowski 2005, van Dalen 2017). Sin embargo, no sabemos si cada fardo funerario fue percibido y tratado como mallqui. La interacción con los muertos se debe documentar en la estratigrafía del fardo, con los episodios de reapertura y reabastecimiento bien visibles (Shimada et al. 2015; van Dalen 2017).

\section{Elaboración del fardo en la cultura Chancay}

Cada paquete funerario o fardo funerario, se compone de diferentes unidades que crean su estratigrafía única. En lugar de capas o elementos de construcción que se esperarían en una excavación tradicional, se trata de capas posteriores de textiles, materiales vegetales, objetos metálicos, pieles de animales y diferentes artefactos. La primera unidad, "la más baja», en esta secuencia es el cuerpo humano momificado o esqueleto, el «núcleo» de cada fardo. El fardo típico de la cultura Chancay se asemeja a una caja rectangular con sus caras ligeramente curvadas, lo que a veces, confiere el aspecto general de un cilindro aplanado. Los fardos estudiados de Cerro Colorado rara vez superan los $120 \mathrm{~cm}$ de longitud, $70 \mathrm{~cm}$ de ancho y 50 $\mathrm{cm}$ de grosor. Cada fardo se compone de un número diferente de varias capas que envuelven el cuerpo creando la secuencia estratigráfica.

\section{La estratigrafía del fardo 28/66/CF 1}

En este artículo presentamos el registro estratigráfico del fardo ubicado en el contexto funerario 1, que se encuentra en la matriz 66 de la Unidad 28, Subunidad 1. La Matriz funeraria 66 tiene $1.40 \mathrm{~m}$ de largo, $100 \mathrm{~m}$ de ancho, y los $0.50 \mathrm{~m}$ de profundidad. El entierro se ubicó a $1.71 \mathrm{~m}$ debajo de la superficie moderna. El fardo estaba acompañado por dos vasijas y una figurina antropomorfa, todas elaboradas en estilo Chancay. La muestra tomada de la tercera capa de algodón (nivel 10B) arrojó la fecha de radiocarbono de $580+/-30$ B.P. (Poz-92420: 1319-1439 d. C. al 95.4\% modelado en $\mathrm{OxCal}$ v. 4.2.3. Usando curvas mixtas de calibración (50:50) IntCal13 y SHCal13 (Hogg et al. 2013; Reimer et al. 2013)), lo que significa que corresponde a finales del Período Intermedio Tardío, casi un siglo antes de la llegada de los Incas a dominar la región. Desenvolver el fardo siempre es una tarea complicada debido a su complejidad interna, así como a la mala

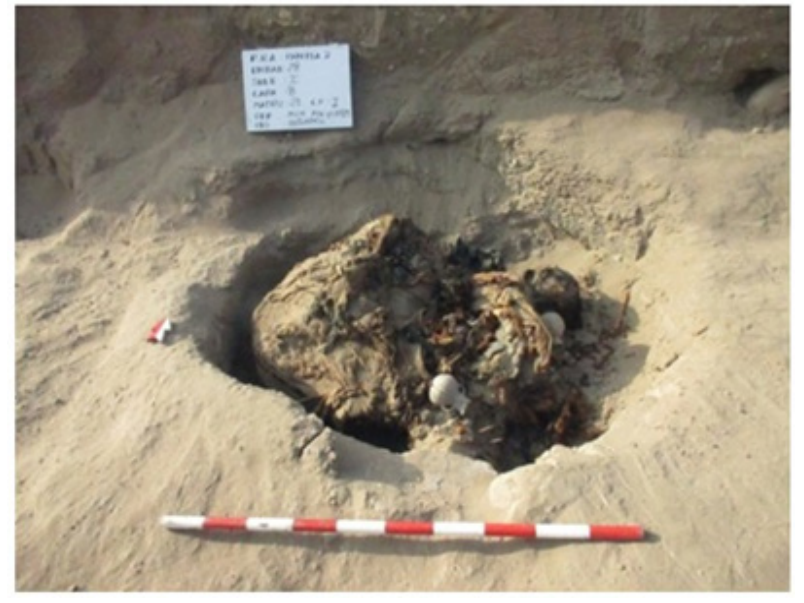

Figura 1: vista panorámica del fardo 28/66/CF 1.

condición de muchos elementos, especialmente los textiles. Utilizamos el método estratigráfico, siguiendo las capas posteriores del fardo, registrando cada elemento individual. El análisis se dividió de acuerdo a los niveles marcados por la aparición de los principales tejidos o capas vegetales que cubrían o envolvían el fardo. Las capas externas del fardo del CF 1 se habían destruido parcialmente, y en su parte media había un agujero (aprox. $15 \times 7 \mathrm{~cm}$ ) hecho por huaqueros, quienes comúnmente insertan largas barras de metal en la tierra para sondear tumbas. Sin embargo, la estratigrafía general permaneció intacta y se presenta a continuación. La mayoría de los textiles fueron tejidos con urdimbre, con técnica de entrelazado de 1 x 1 . Para aquellos que no lo fueron, la diferencia se especifica en la descripción.

Nivel 1. Las capas externas, severamente dañadas (figura 2). Los elementos:

- Textil 1: conservado solo en fragmentos, este es un manto del color blanco-beige. Un fragmento, posiblemente uno de los extremos, está unido a la otra parte del tejido con un cordón.

- Capa de algodón, espesor de 3 a $5 \mathrm{~cm}$, colocada alrededor del fardo.

- Textil 2: fragmento mal conservado de un manto más grande. El color básico es azul oscuro, con bandas creadas por urdimbres de hilo marrón. Este textil fue colocado en el lado anterior superior del haz.

- Textil 3: manto pequeño, color base marrón con bandas de figuras geométricas creadas con ur- 
dimbres de hilo azul y beige complementarios. Colocado debajo del textil 2.

- Textil 4: creado a partir de al menos cuatro mantos más pequeńos cosidos juntos. El color original era el blanco, con la superficie ligeramente abierta. Envuelve todo el fardo.

Nivel 2. Se refiere al textil 6, con una superficie ligeramente abierta, compuesta por dos mantos cosidos horizontalmente y envueltos alrededor del fardo. El color original era el beige (figura 3).

En la parte anterior superior había cinco láminas de cobre, colocadas en el lugar correspondiente a la cabeza del individuo. Tres de ellas estaban en la superficie del textil 6 y las dos últimas directamente debajo. Su deposición fue aparentemente simultánea a la del textil.

Nivel 3. Se refiere al elemento principal, el textil 7, y al grupo de elementos colocados en la parte superior del fardo (figura 4):

- Capa de algodón.

- Textil 5: manto pequeño, con cordones unidos a sus esquinas, color original beige o blanco.

- Textil 8: manto pequeño, tejido $2 \times 1$, con urdimbre, color original blanco o beige.

El Textil 7 fue el manto que originalmente envolvió todo el fardo. En el momento del estudio solo quedaba el $40 \%$, y el color original se había perdido. Había una pequeña lámina de cobre que estaba sujeta con un cordón a este textil, en una parte inferior del fardo. Por abajo estaba el textil 9 (Figura 5) un manto con la superficie dividida en bandas marrones y blancas de diferentes anchos, hechas de hilos alternativos de estos colores utilizados como urdimbres. Este textil envolvía el fardo, salvo su parte superior.

Nivel 4. En este nivel, se registró el textil 10 (figura 6). Este textil no se estiró y tenía muchas arrugas, y se conservó en fragmentos, aunque fue posible determinar que originalmente envolvió todo el fardo. Había una lámina de cobre rectangular en una de las arrugas, en la parte superior del fardo.

Nivel 5. El textil 11 ocupó otra posición en la secuencia estratigráfica (Figura 7). Su superficie estaba decorada con dos colores diferentes de urdimbre, con bandas dobles de color marrón separadas a intervalos regulares por bandas de color beige. Este textil envolvió todo el fardo de una manera «envolvente», con sus esquinas atadas en un nudo en el lado central anterior del fardo. Había tres hojas de cobre en un pliegue superior en el manto. Todos ellos eran rectangulares, con los bordes ligeramente cóncavos. El dobladillo de la tela en ese lugar tenía una pequeña cuenta de cobre unida con un cordón. Una parte importante del textil 11 estaba en estado de descomposición, con las urdimbres beige desgastadas.

Nivel 6. Debajo del material textil 11 había una capa de algodón de 3 a $6 \mathrm{~cm}$ de grosor (figura 8). Envolvió el fardo a excepción de su extremo superior, donde otro elemento, el textil 12 , ya era visible.

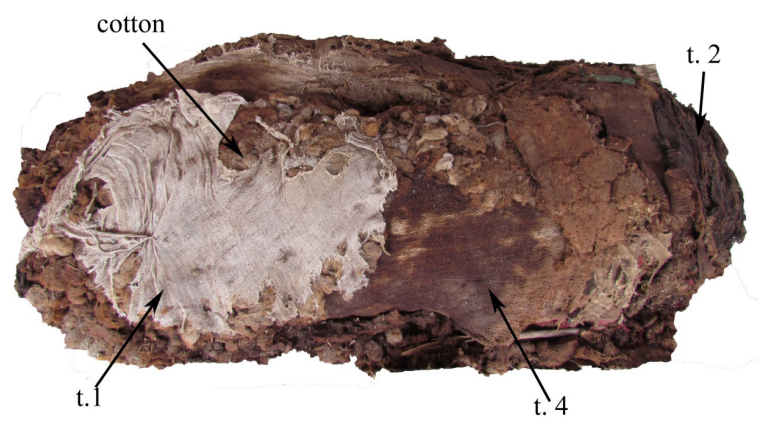

Figura 2. Nivel 1.

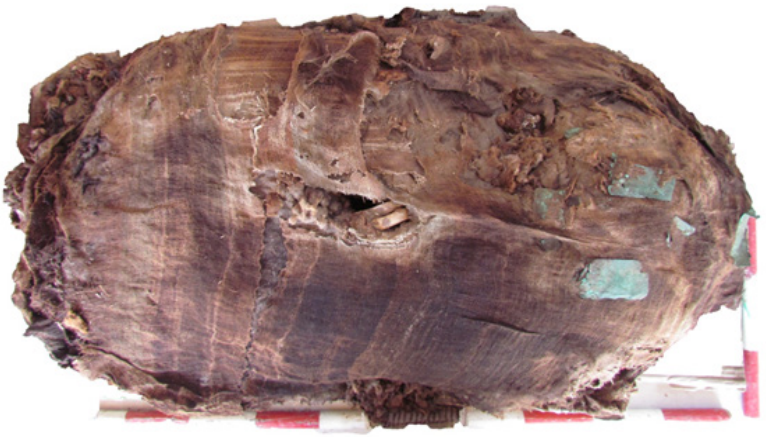

Figura 3. Nivel 2.

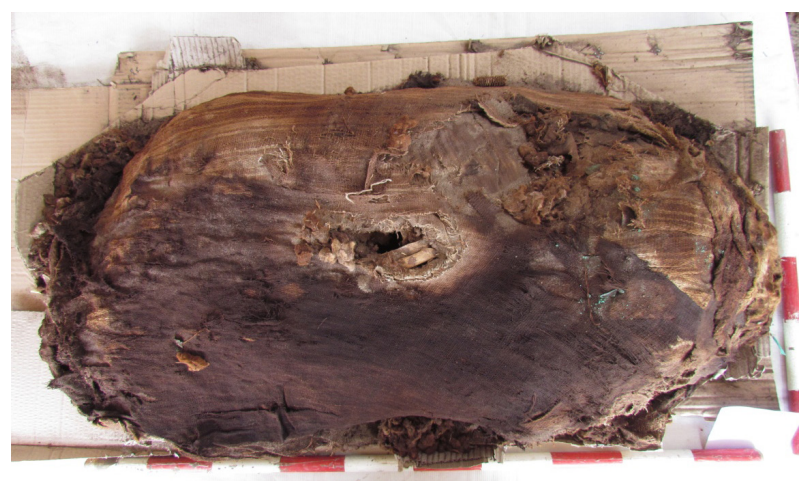

Figura 4. Nivel 3. 


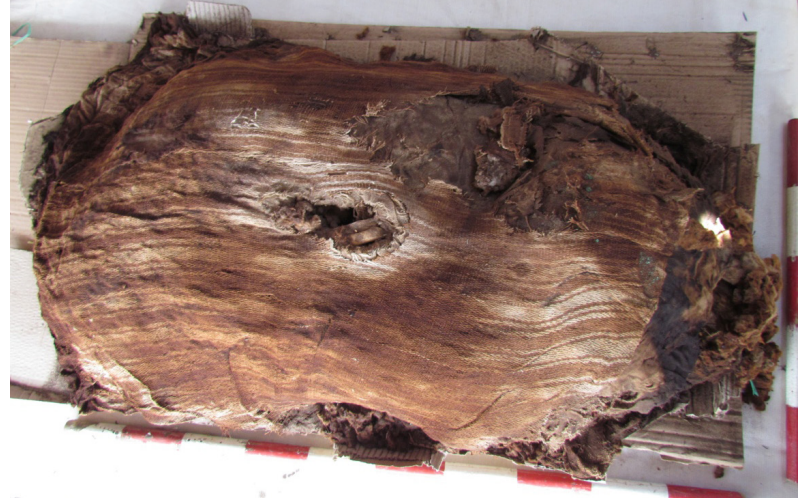

Figura 5. Nivel 3, textil 9.

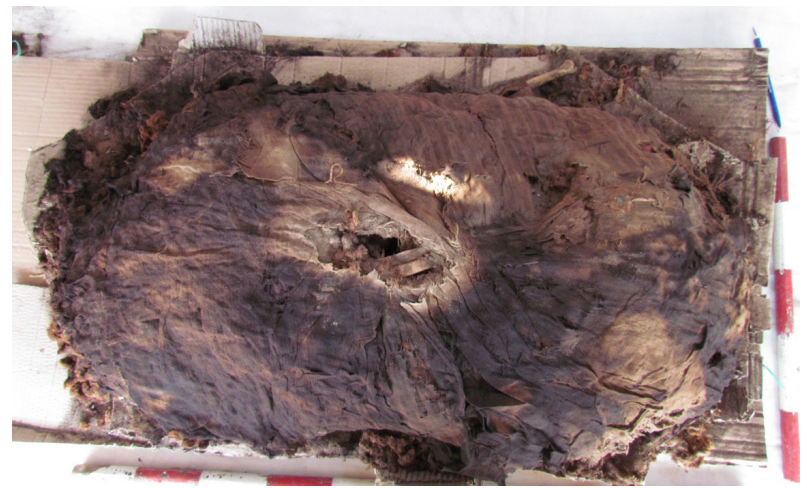

Figura 6. Nivel 4.

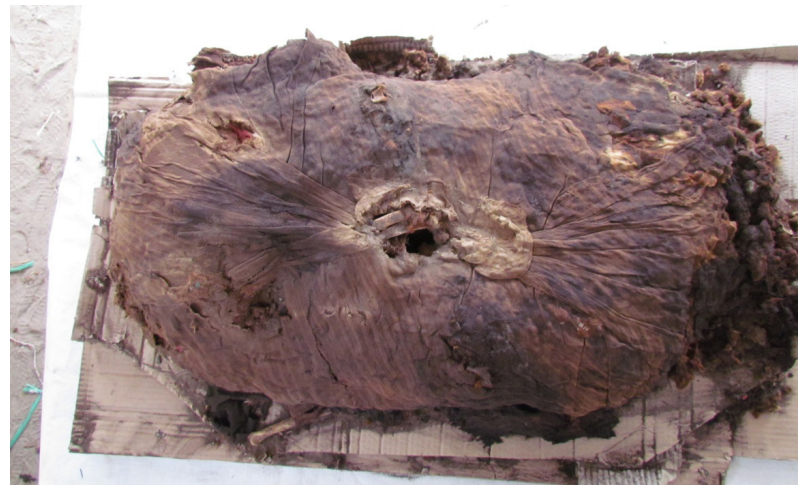

Figura 7. Nivel 5.

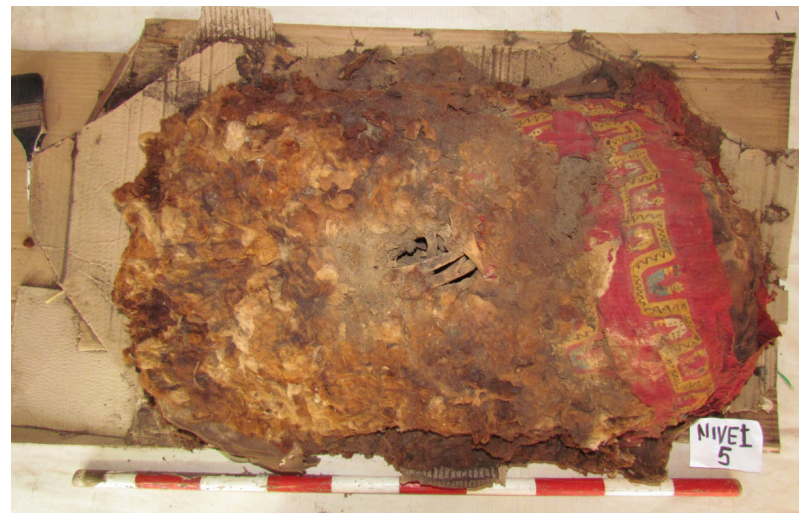

Figura 8. Nivel 6.

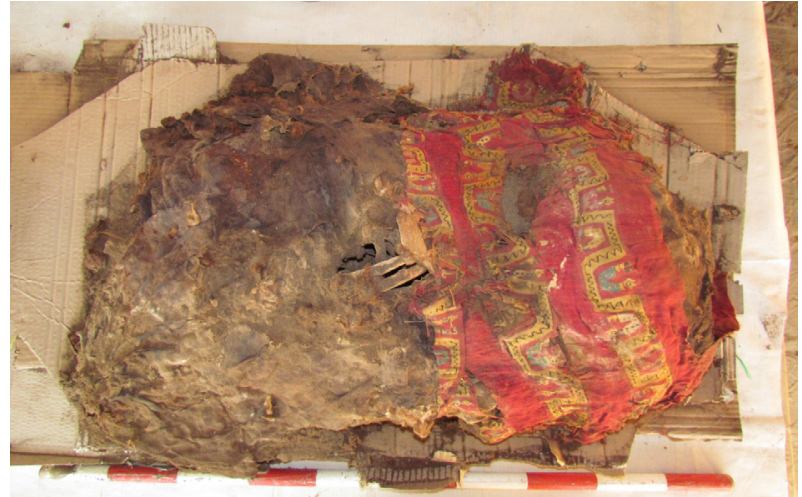

Figura 9. Nivel 7A.

Nivel 7A. En este nivel había dos capas: textiles 12 y 13 , ambos en contacto físico con la capa de algodón del nivel 6 (figura 9). La materia textil 12 envolvió solamente la parte superior del fardo. Era un manto cosido de al menos ocho bandas horizontales, cada una hecha de lana de camélido en la forma de cara de trama, con urdimbres amarillas gruesas y finas tramas rosadas, y con superficie compacta (urdidos invisibles). Aparte de un caso en el que se cosieron dos bandas sin decorar, cada segunda banda había sido decorada. Tenía la forma de una figura de tipo meandro (curvilínea) que continúa de un borde a otro, y en cada flexión hay una figura que representa a una criatura con forma de serpiente doblada a mitad del cuerpo (figura 19). Tanto el adorno de tipo meandro como las figuras de animales se habían ejecutado utilizando tapices y tapices excéntricos, y en algunas partes también en tapices de hendidura. En este último caso, las partes flotantes habían sido sembradas en la parte principal del textil (la parte roja). Juntos, había cuatro bandas decoradas y cinco sin decoración. Aunque el textil no se conservó en su totalidad, su posición en el fardo sugiere que las dimensiones originales no diferían notablemente de las actuales.

Nivel 7B. En este nivel, el textil 13 se registró en su totalidad (figura 10). Tenía decoración en forma de bandas verticales de color marrón y beige (urdimbres) colocadas alternativamente. Este textil estaba en mal estado, desgarrado y descompuesto en muchos lugares; sin embargo, fue posible afirmar que originalmente se había envuelto alrededor del fardo. Debajo del textil 13 había otro, textil 14, de color marrón con una superficie abierta. Este textil originalmente envolvió el fardo, pero solo quedaban pequeños frag- 
mentos en el momento del examen. Debajo del textil 14 había un pequeño manto colocado en la parte superior del fardo. Este textil 16 estaba decorado con bandas dobles de color marrón (divididas por una línea delgada de color beige), separadas a intervalos regulares por bandas de color beige. Debajo de ese pequeño manto estaba el textil 15 , un gran manto decorado con bandas alternas de color marrón claro y oscuro (color original desconocido). Las bandas oscuras tenían rectángulos blancos como decoración adicional. Este textil había sido cosido de al menos tres piezas, con costuras verticales, probablemente se había envuelto alrededor de todo el fardo.

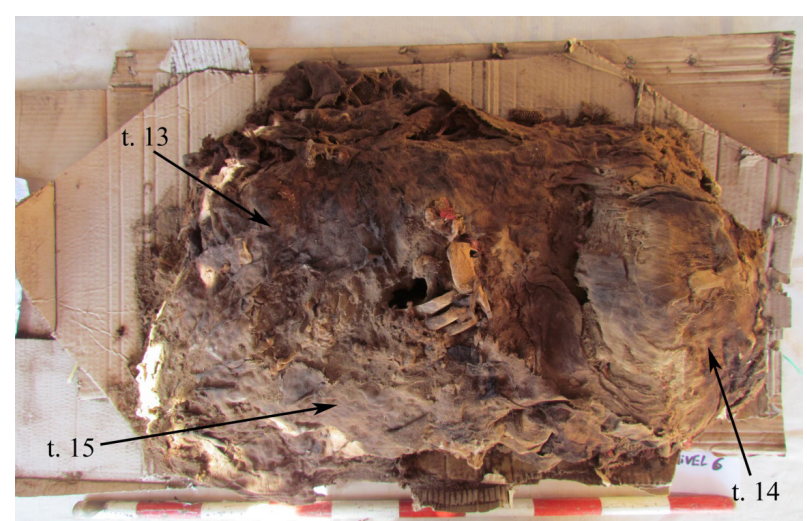

Figura 10. Nivel 7B.

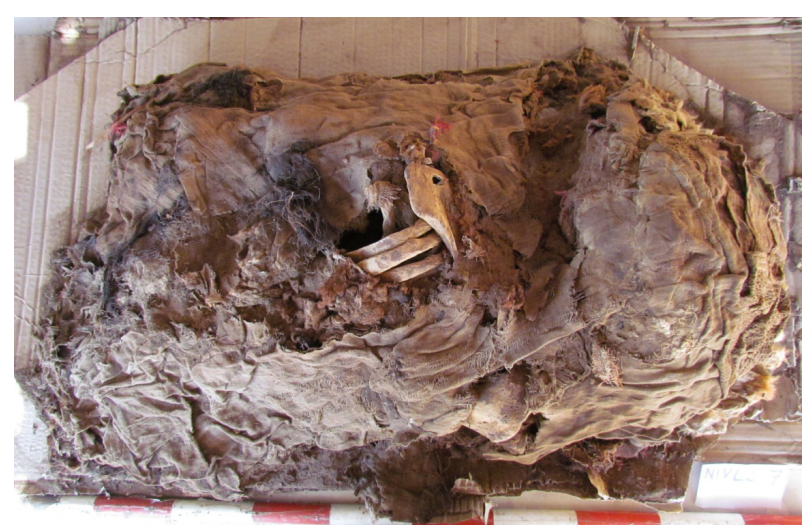

Figura 11. Nivel 8.

Nivel 8. Después de quitar el textil 15, otras dos piezas fueron visibles (figura 11). El textil 17 era un manto doble con superficie abierta, de color marrón por descomposición (se desconoce el color original). Este elemento se había colocado en el fardo de manera similar a un chal, con ambos extremos más abajo, en el área "torácica». El segundo textil, 18, se colocó debajo del textil 17 y se envolvió alrededor del fardo (figura 12). Este carecía de decoración y estaba mal conservado, aunque el color beige original todavía era visible en algunos lugares.

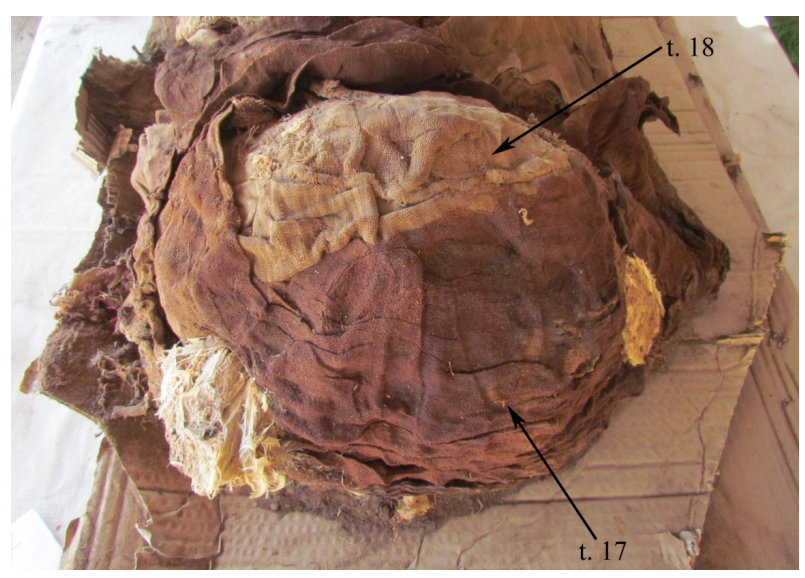

Figura 12. Nivel 8, parte superior del fardo.

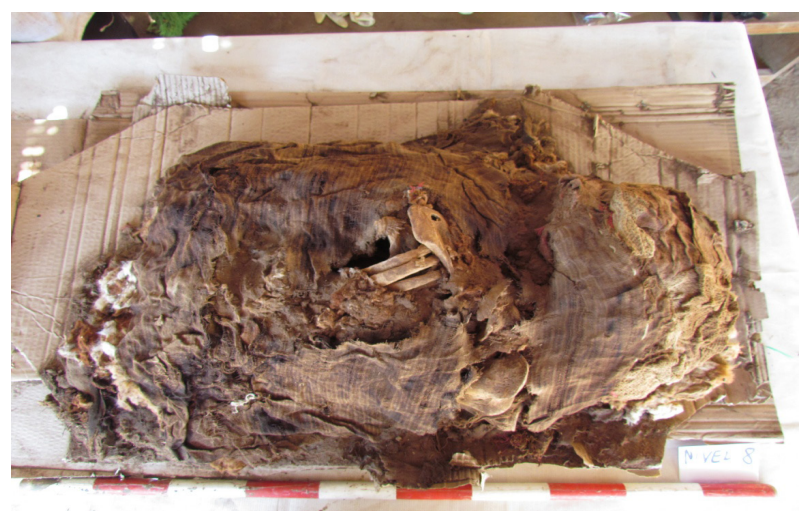

Figura 13. Nivel 9.

Nivel 9. En este nivel registramos pocos textiles diferentes (figura 13). El primero, textil 19, era un manto decorado en estilo típico con bandas de por lo menos dos colores diferentes (no diferenciados por mala conservación) colocadas alternativamente; y se envolvió horizontalmente alrededor del fardo, excepto su parte superior.

Nivel 10A. Se refiere al nivel debajo del textil 19, donde era visible un grupo de pequeños mantos envueltos alrededor de la parte superior, al igual que una capa de algodón que cubría la otra parte del fardo (figura 14). Este nivel incluía:

- Textil 20: una gasa beige.

- Textil 21: un manto beige-pardo, con los bordes decorados con una hilera de aves marinas representadas en el perfil, ejecutadas utilizando la técnica de brocado con hilos suplementarios 
rosado. Como en los otros casos, esta pieza estaba fragmentada.

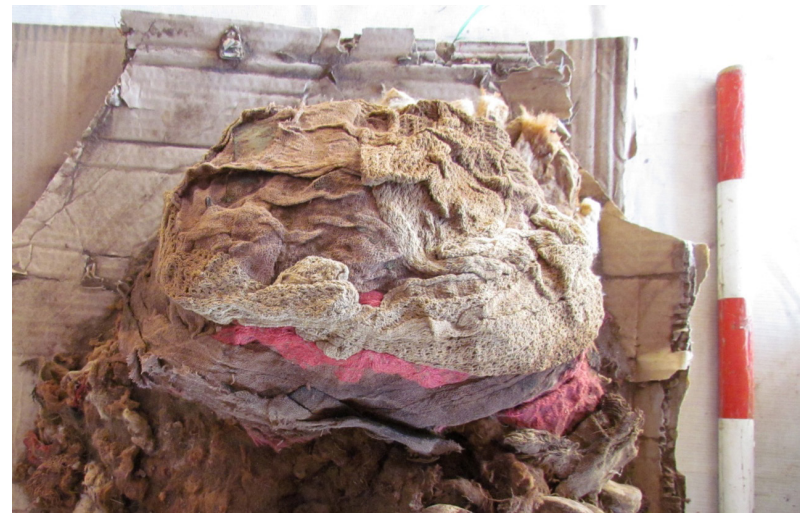

Figura 14. Nivel $10 \mathrm{~A}$.

Debajo del textil 21 había tres láminas de cobre (figura 15). Dos de ellos eran rectangulares con bordes cóncavos y yacían uno al lado del otro. El tercero tenía clara forma rectangular y estaba abajo. Las piezas de metal estaban en el textil 22, el pequeño manto que envolvía la parte superior del fardo a manera de un pañuelo en la cabeza. Este textil se elaboró de manera deformada, con patrones de entrelazado de $1 / 2$ y $2 / 2$ mixtos. Originalmente, este textil tenía dos colores, con el beige como el color básico y las bandas horizontales azules creadas con tramas teñidas. Debido a la descomposición, la mayoría del textil 22 había perdido sus colores originales, por lo que era imposible determinar exactamente cómo se había decorado.

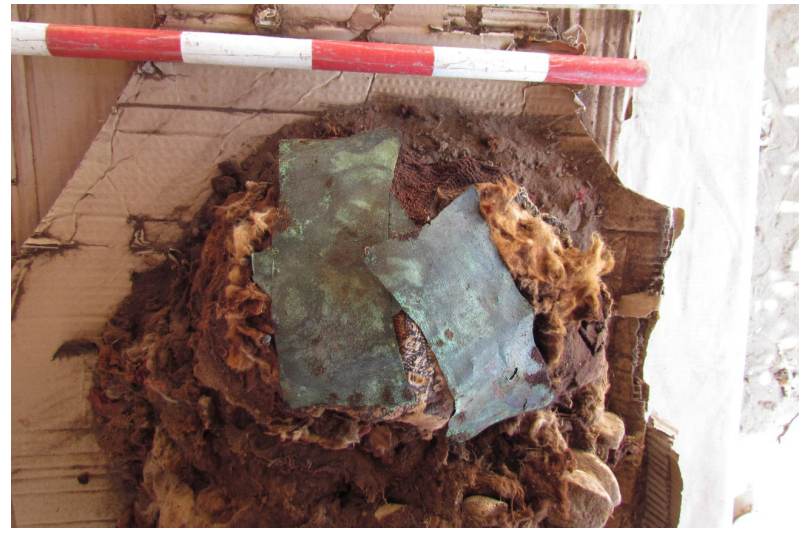

Figura 15. Nivel $10 \mathrm{~A}$, láminas de cobre.

Nivel 10B. En este nivel, se registró una capa de algodón de $5 \mathrm{~cm}$ de grosor (figura 16). Esta capa se encontraba debajo del textil 19 y el textil 22, y se envolvió alrededor del fardo entero. La excepción fue la parte superior, donde la cantidad de material vegetal fue notablemente menor. El textil 22 estaba en contacto físico con los textiles 23 y 24 , y con la cabeza del cadáver.

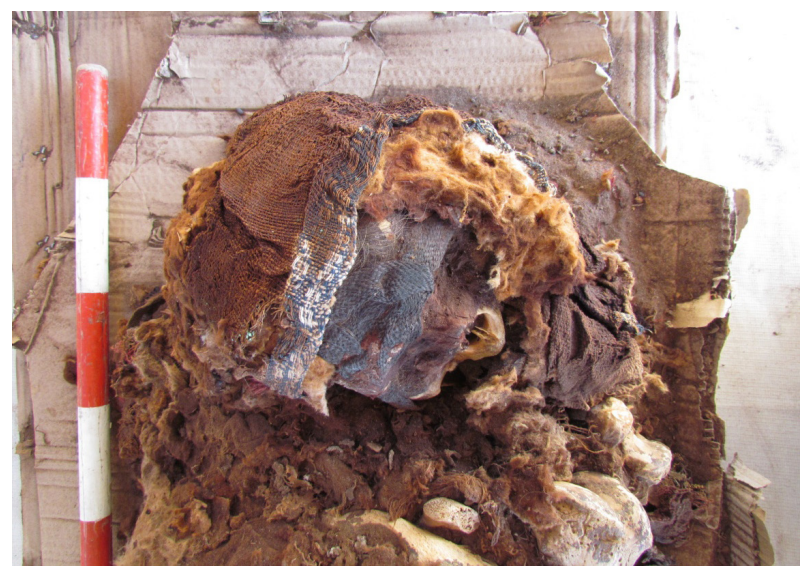

Figura 16. Nivel 10B.

Nivel 11A. En este nivel, apareció el cuerpo esqueleto del individuo (figura 17). En la parte posterior del cráneo había el textil 24, una pequeña pieza marrón (color original desconocido) entre el algodón y el textil 22 por un lado, y entre el textil 23 y el cráneo por el otro. La cabeza estaba envuelta con una banda azul oscura o llautu, anudada en la frente (textil 23). El resto del cuerpo estaba cubierto con fragmentos de tejido 25, tan mal conservados que era imposible especificar si había sido la ropa del cadáver o el manto que envolvía el cuerpo.

Nivel 11B. Este nivel (figura 18) se refiere al esqueleto expuesto, visible en aquellas áreas que se retiraron todos los textiles (en cuanto se pudo) para no alterar su posición anatómica (por ese motivo, el textil 23 todavía estaba presente, ya que era imposible retirarlo sin que también perturbara el cráneo). Uno puede notar que hay muchos pequeños fragmentos textiles y piezas de algodón entre los huesos. Esto se debió al agujero hecho por los huaqueros, en el que habían caído diferentes fragmentos de las capas superiores. También hay elementos de cada capa visibles debajo del esqueleto. Como en la mayoría de los casos, su estado de conservación era mucho peor que en la parte anterior del fardo, y muchos de ellos se encontraron pulverizados. 


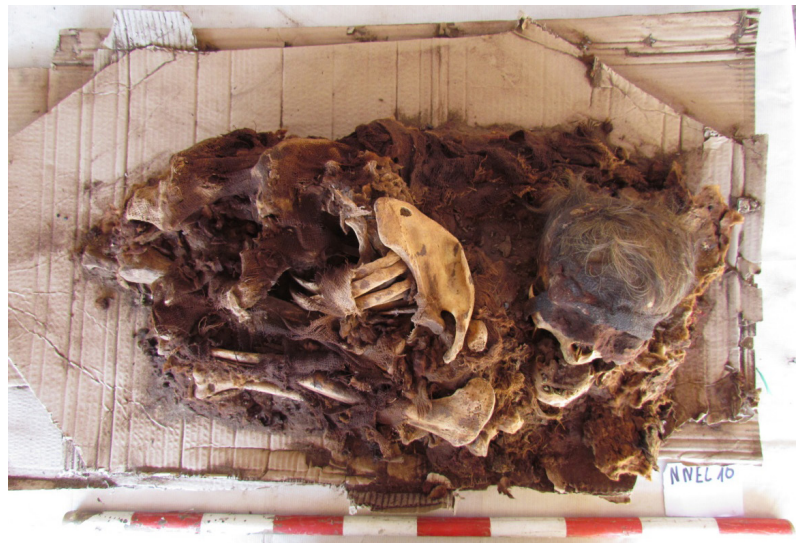

Figura 17. Nivel 11A.

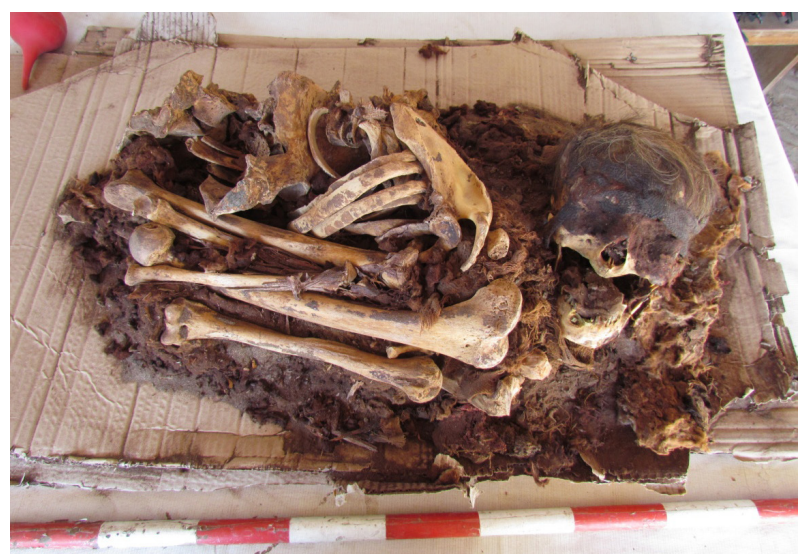

Figura 18. Nivel 11B.

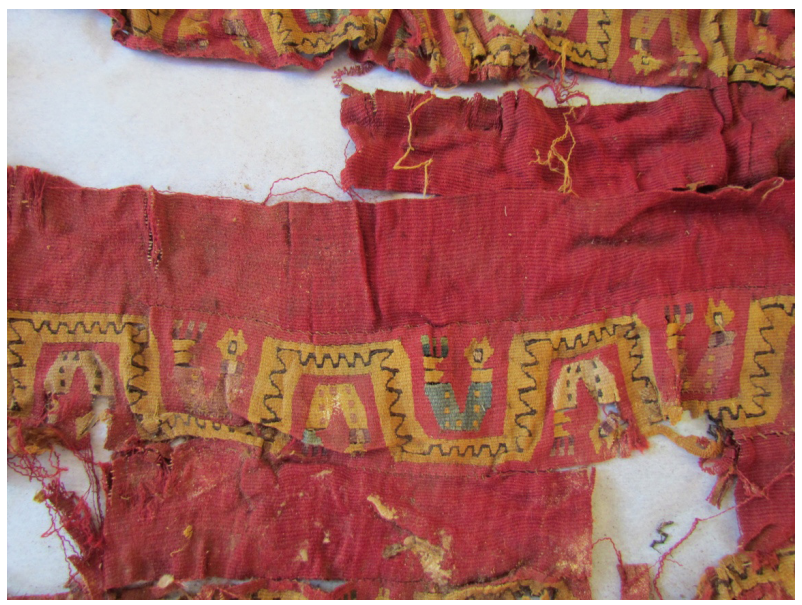

Figura 19. Textil 12, detalle de la decoración.

El cadáver estaba acostado sobre su espalda y parcialmente en el lado izquierdo, en una posición flexionada con los muslos aducidos al abdomen. Las extremidades superiores se flexionaron y las manos se colocaron en el tórax. El cráneo se giró hacia la dere- cha y se inclinó hacia abajo, lo que podría haber sido un acto intencional o podría haber ocurrido accidentalmente una vez que se hizo el fardo. La mayor parte de los tejidos blandos estaban descompuestos, aunque algunos pedazos de piel aún estaban adheridos a los huesos. Se bajó la mandíbula y se colocó un trozo de algodón dentro de la cavidad bucal. El algodón se había usado para cubrir la cara de los muertos, y después de la descomposición del tejido blando también cayó dentro de las órbitas. También había manchas verdes en los huesos de la cara, lo que indica la presencia original de algún adorno de metal colocado directamente sobre la piel. Las costillas y las escápulas se desplazaron hacia la pelvis, lo que probablemente ocurrió después del entierro por el peso del fardo y la presión externa.

\section{El cadáver}

El cadáver era un hombre de 50 a 60 años (la edad de muerte se estableció de acuerdo con Buskistra, Ubelaker 1994) con una deformación craneal erecta tabular típica de tipo costero. En el techo orbital se observó cribra orbitalia (segundo grado). La presencia de estos cambios sugiere anemia por deficiencia de hierro, una dieta deficiente en vitamina B12 o infecciones parasitarias (Hengen 1971; Walker et al. 2009). Todos los dientes maxilares se habían perdido durante la vida. La dentición mandibular se conservó parcialmente. Desde la parte izquierda del arco dental todavía estaban presentes el segundo incisivo, el canino y el segundo premolar. En el lado derecho notamos la presencia del primer y segundo premolares y el primer molar. En la corona del primer molar había caries dental gruesa, y en las superficies de contacto de los premolares había lesiones de caries más pequeñas. Es muy probable que los ejemplos restantes examinados hayan sobrevivido a algunas inflamaciones graves que terminaron con fístulas graves en el alvéolo de la parte superior izquierda incisiva y la parte inferior izquierda del canino. Como la mayoría de los adultos enterrados en la necrópolis de Cerro Colorado, padecía enfermedades periodontales. Además, la corona del segundo incisivo izquierdo se había roto durante la vida. La frecuencia de fracturas de dientes ante mortem en esta población es muy alta. Observamos 
que los pescadores tradicionales modernos abren las conchas de moluscos directamente con sus dientes, lo que influye en la condición dental general. Esta podría ser la explicación del dramático estado de la dentición en los restos del cadáver estudiado.

Se observaron numerosos cambios en la columna vertebral. En la parte torácica inferior y en la parte completa de la vértebra lumbar, había osteofitos bien desarrollados en la parte anterior de los cuerpos vertebrales. La quinta vértebra lumbar y el hueso sacro se fusionaron en ambos lados, pero los cuerpos no estaban conectados, lo que significa la sacralización parcial de la L5. El segmento de Th11 a L1, en su lado anterior, se conectó mediante osificación masiva del ligamento anterior longitudinal. Las etapas iniciales de la osificación de los ligamentos también se observaron en las otras vértebras. La osificación de los ligamentos amarillos se observó a lo largo de toda la longitud de la parte torácica de la columna vertebral. La presencia del ligamento anterior longitudinal osificado puede indicar la aparición de una enfermedad conocida como DISH (Ortner 2003). Las articulaciones de las extremidades superiores e inferiores, especialmente las articulaciones del hombro y la rodilla, presentaron deformaciones articulares típicas de la enfermedad articular degenerativa.

\section{La fabricación del fardo}

Aunque la mayoría de los fardos contenían restos momificados o semi-momificados, el cuerpo del individuo del fardo 28/66/CF 1 estaba esqueletizado. Esto puede significar que el cuerpo no fue sometido al embalsamamiento, o que la aplicación de bálsamo no detuvo la descomposición. Sin embargo, todavía estamos trabajando en el problema de si la gente Chancay practicó una momificación artificial. Como los tejidos blandos del individuo se habían deteriorado, no pudimos reconocer si su cuerpo recibió algún tratamiento especial (por ejemplo, pintura).

Lo que sabemos es que los dolientes colocaron en la cara algunos objetos de cobre, como lo sugieren las manchas verdes en los huesos faciales, y luego cubrieron la cabeza o incluso todo el cuerpo con algodón. Luego llenaron la cavidad bucal con pequeñas láminas de cobre y algodón, y ataron el llautu o el textil 23 alrededor de la cabeza. Todas estas actividades po- drían haber ocurrido inmediatamente después de la muerte, o cuando se estaba preparando el fardo.

Durante la fabricación del fardo, el cuerpo todavía estaba en una sola pieza, como lo indica el descubrimiento de casi todos los huesos en sus posiciones anatómicas correctas. Por lo tanto, el individuo se colocó (probablemente desnudo) en la posición fetal y se envolvió en la primera capa: textil 25, con el textil 24 debajo de la parte posterior de la cabeza. Luego, los fabricantes del fardo colocaron una capa de algodón alrededor del fardo y envolvieron el textil 22 alrededor de la parte superior, colocando en su superficie tres láminas de cobre, y por encima de estos los textiles $21 \mathrm{y}$ 20. Luego se agregaron los textiles posteriores. Algunos de ellos sirvieron como protectores y se usaron para envolver todo el paquete, o su parte más grande: textiles $19,18,15,14,13,12,11,10,9,6,4,2$ y 1 . Parece probable que los participantes de la ceremonia primero colocaron el textil en el suelo y luego lo colocaron sobre él. Mientras envolvían el fardo con los principales textiles, también colocaban grupos de pequeños mantos alrededor de su parte superior $(22,21$ y $20,17,16$, luego 8 y 5, luego 3 y 2). Esta actividad podría estar relacionada con la colocación de "cabezas falsas» en la parte superior de los fardos, una costumbre conocida de muchos cementerios de la cultura Chancay, como Macatón (van Dalen; 2013). También había cuatro capas de algodón entre los grupos de dos, cuatro o cinco tejidos grandes de tipo sudario, y también justo debajo del textil 1. El textil muy especial 12, el tapiz, se colocó en la parte superior anterior del fardo. Durante la confección del paquete, los participantes colocaron pequeñas láminas de cobre cortadas de algunas piezas más grandes en los pliegues de los mantos o entre ellas. Solo podemos suponer que la ceremonia de la confección de fardos se asemejaba de alguna manera a la descrita por Bartolomé de las Casas. Así, el equipo de fabricantes de fardos estaría compuesto por diferentes grupos de dolientes, cada uno de los cuales colocó una capa (o capas) mientras realizaban su parte del ritual. Todo el proceso duraría de cinco a diez días, tal vez incluso más tiempo.

\section{Discusión}

El individuo del fardo 28/66/CF 1 murió a los 50 o 60 años de edad. Este lapso de vida fue bastante 
típico de los pescadores, cuyos restos fueron enterrados en el sitio de Cerro Colorado, mientras que las otras personas, incluidos los artesanos, generalmente murieron en la cuarta o, con menos frecuencia, la quinta década de la vida. La osteobiografía del cadáver encaja en el estado general de la población de Cerro Colorado, especialmente en el tema de la dentición, ya que la inflamación que resulta en fístulas severas es extremadamente común. Lo mismo puede decirse acerca de la enfermedad degenerativa de la articulación (DJD), así como las malformaciones de la columna vertebral. Dicha osteofitosis severa y la osificación de los ligamentos fueron problemas comunes en esa área, y la enfermedad DISH ocurrió esporádicamente.

En nuestra opinión, los cambios degenerativos, como la enfermedad degenerativa de las articulaciones, la osteofitosis grave y la osificación de los ligamentos son pruebas de que el individuo realizó un trabajo físico intenso, como lo hacen los pescadores modernos tradicionales. A medida que se formaba hueso nuevo en sus canales auditivos, estaba perdiendo la audición y probablemente era sordo en los últimos años de su vida. Las fístulas en el alvéolo del incisivo superior izquierdo y del canino inferior izquierdo indican inflamación prolongada, tal vez incluso necrosis. Esto significa que el hombre tenía un dolor constante durante los últimos meses o incluso años de su vida. En la tradición andina, la enfermedad es, en muchos casos, el resultado de una distorsión en el equilibrio natural de las fuerzas que impulsan el universo. Esta distorsión puede ocurrir debido al pecado (incluso contra seres no materiales). La actividad de poderes naturales como el sol o el viento, las fuerzas sobrenaturales, el contacto con los muertos y la brujería también pueden causar enfermedades en esta tradición (Marsteller et al. 2011; Altamirano 2018). Se desconoce, sin embargo, cómo exactamente la gente de Cerro Colorado percibió los problemas de salud que afectaron al hombre del fardo 28/66/CF 1. Las malformaciones de los dientes y la columna vertebral eran comunes entre ellos. En el caso de los pescadores, la exostosis era demasiado. La pregunta de cómo explicaron la presencia de estas condiciones sigue sin respuesta.

El cadáver que iba a ser enterrado dentro del fardo era el de un miembro de la comunidad de pescadores. Este supuesto se basa en la presencia de la formación de hueso nuevo (exostosis) en ambos canales auditivos. Los estudios clínicos han demostrado que estos tumores crecen como resultado del buceo frecuente en aguas frías (Reimers et al. 2008). Según Rostworowski, la sociedad costera de los Andes Centrales se dividió, durante los tiempos de los incas, en agricultores, mercaderes especialistas, artesanos y pescadores, que vivían en distritos asentamientos separados. Los pescadores, de la zona litoral, hablaban su propio idioma y adoraban a las deidades y los fenómenos naturales asociados con los recursos marinos (Rostworowski 2005: 117-152). Todavía no está claro, sin embargo, si en el Período Intermedio Tardío las personas vivían de una manera similar.

En el sitio de Cerro Colorado, tanto pescadores como artesanos fueron enterrados uno al lado del otro. Esto puede significar que la división descrita por Rostworowski no existió en los siglos xiii y xiv, o que estos grupos vivieron separados, pero usaron el mismo cementerio. En resumen, sabemos que los restos son los de un pescador que realizó un trabajo físico intenso. Sufría dolorosos síntomas dentales y de columna, así como la presencia de parásitos, anemia o deficiencia de vitamina B12. Cada una de estas condiciones podría haber causado debilidad física y quizás algunos problemas psiquiátricos (en caso de deficiencia de vitamina B12). Sin embargo, sus parientes trataron el cuerpo con gran respeto, utilizando un número significativo de textiles para crear el fardo, incluido el tapiz (textil 12). Este último podría simplemente referirse a sus habilidades de pesca (si aceptamos que su iconografía representa el mar y seres marinos), o tal vez estaba destinado a ayudarlo en su viaje después de la muerte. La cantidad significativa de trabajo puesto en la creación de su fardo sugiere que era una persona especial, por alguna razón. Podría haber tenido habilidades de pesca sobresalientes, y tal vez eventualmente llegar a ser el líder de los pescadores con los que trabajó, cumpliendo también con alguna función política. ¿Podría ser posible que después de la muerte fuera adorado como un antepasado divino o mallqui? En el sitio descubrimos dos fardos en los que se pueden haber colocado nuevos textiles en un fardo ya cerrado; la estratigrafía sugiere que esto podría suceder algún tiempo después de la deposición original del fardo, y esto se verificará con la datación por radiocarbono. El fardo28/66/CF 1 , sin embargo, no presenta ningún signo de reaper- 
tura o reestructuración, y parece ser el producto de un evento único. No había rastros visibles de que la tumba que se haya reabierto, excepto los que dejaron los huaqueros modernos. Por lo tanto, no hay signos de interacción entre los muertos y sus parientes vivos. Eso significaría que el individuo descrito en este artículo no estaba destinado a convertirse en mallqui; sin embargo, fue enterrado dentro de un fardo funerario. Las perspectivas percibidas de los muertos entre la sociedad del Período Intermedio Tardío fueron probablemente más complicadas que la simple división de antepasados y no ancestros. Tal vez la versión costera del culto a los antepasados no requería interacción con los muertos, como sugiere Isbell (Isbell 1997: 144. Sin embargo, véase Shimada et al. 2016).

\section{Conclusión}

El estudio estratigráfico de los fardos funerarios de Chancay revela la complejidad del patrón funerario entre las últimas sociedades costeras prehispánicas. En este artículo presentamos el caso de los restos de un pescador de 50 a 60 ańos de edad, que al final de su vida sufrió dolores dentales y afecciones de la columna vertebral, y también era sordo. Durante la ceremonia fúnebre, su cuerpo fue envuelto en 25 tejidos diferentes, así como algunas capas adicionales de algodón, luego fue enterrado en el sitio de Cerro Colorado. Todos los textiles eran mantos con caras de urdimbre de diferentes dimensiones, hechos de fibras de algodón. La única excepción fue el textil 12, el tapiz decorado con el adorno tipo meandro y con los seres con forma de serpiente. Este manto estaba hecho de lana de camélido. El diseño general del paquete podría estar condicionado por la biografía del hombre antes de la muerte, incluida la posición social que ocupaba. Los documentos coloniales sugieren que este tipo de tratamiento estaba reservado solo para algunas personas importantes, percibidas como ancestros poderosos que poseían poder generativo. Sin embargo, la estratigrafía del fardo28/66/CF 1 no presenta rastros de una mayor interacción con los muertos. Estamos tratando entonces con una tradición funeraria de alguna manera distinta, que, con suerte, será entendida en el futuro.

\section{Agradecimientos}

Un agradecimiento y reconocimiento a todas aquellas instituciones que han hecho posible la presente investigación, mediante su financiamiento: A las Municipalidades Provincial de Huaura, Distrital de Santa María, al Gobierno Regional de Lima, a las Asociaciones de Vivienda Los Pinos (I, III, IV y V Etapa). Al Sr. Elías Mota, presidente de la I Etapa de Los Pinos. De igual manera, a los más de 200 arqueólogos que han participado en los trabajos de excavación y gabinete, principalmente al arqueólogo Hans Grados Rodríguez (Jefe de Campo). La datación por radiocarbono fue apoyada por el Centro Nacional de Ciencias en Polonia (número de concesión 2015/19 / N / HS3 / 00552). Los autores desean agradecer a Justyna Marchewka y Andrzej Krzanowski por sus valiosos comentarios sobre el análisis. 


\section{Bibliografía}

Altamirano, Alfredo (2018). «Onccoy: las enfermedades en el mundo mochica». En: Boletín de Lima, 186: 4980. Lima.

Arriaga, Pablo (1968 [1621]). Extirpación de la idolatria del Piru. Madrid: Ediciones Atlas.

Bastiand, María (2000). Producción textil prehispánica. Investigaciones Sociales, 5: 125-144. Instituto de Investigaciones Histórico Sociales, Universidad Nacional Mayor de San Marcos. Lima.

Buikstra, Jane \& Dennis Ubelaker (1994). «Standards for data collection from human skeletal remains». En: Research series No. 44. Fayetteville, Arkansas: Arkansas archeological survey research series 44 .

Casas, Bartolomé de las (1892 [1550]). De las antiguas gentes del Perú. Lima.

Cieza de León, Pedro (1946 [1553]). La cronica del Peru. Primera parte. México: Editorial Nueva Espana.

Сово, Bernabé (1964 [1653]). Historia del Nuevo Mundo. Madrid: Ediciones Atlas.

Fung, Rosa (1960). Huaral: inventario de una tumba saqueada. Etnología y arqueología, 1: 74-129. Universidad Nacional Mayor de San Marcos. Lima.

Hengen, O. P. (1971). Cribra orbitalia pathogenesis and probable etiology. Homo, 22:57-76.

Hogg, A. G.; Q. Hua, P.g. Blackwell, M. Niu, C. E. Buck, T. P. Guilderson, T. J. Heaton, J. G. Palmer, P. J. Reimer, J. W. Reimer, C. S. M Turney, \& S. R.H. Zimmerman (2013). SHCal13 Southern Hemisphere Calibration, 0-50,000 Years cal BP. Radiocarbon, 55(4). 1889-1903.

Holquin, Gonzalo (2007 [1608]). Vocabulario de la Lengva General de todo el Perv llamada Lengva Qquichua o del Inca. Digitalizado por Runasimipi Qespisqa Software(http://www.runasimipi.org) para publicación en el internet, 2007.

IsbeLL, William (1997). Mummies and Mortuary Monuments. A Post processual Prehistory of Central Andean Social Organization. Texas University Press.

Kaulicke, Peter (2001). Memoria y muerte en el Perú Antiguo. Fondo Editorial PUCP.

KrZanowski, Andrzej (1991). Estudios sobre la Cultura Chancay. Universidad Jaguelona.

KrZANowski, Andrzej (2008). Kultura Chancay: środkowe wybrzeże Peru uschytkuepokiprekolumbijskiej (X-XVIw.). Krakow-Warszawa: Instytut Amerykanistyki i Studiow Polonijnych Uniwersytetu Jagiellońskiego, PTSL.
Makowski, Krzysztof (2005). Deificación frente a ancestralización del gobernante en el Perú prehispánico: Sipán y Paracas. En: Arqueologia, geografia e historia: aportes peruanos en el 50 ${ }^{\circ}$. Congreso de Americanistas. VarsoviaPolonia. 2000: 39 - 80. Fondo Editorial PUCP.

Marsteller, Sarah, C. Torres-Rouff \& K. J. Knudson (2011). Pre-Columbian Andean sickness ideology and the social experience of leishmaniasis: A contextualized analysis of bioarchaeological and paleopathological data from San Pedro de Atacama, Chile. International Journal of Paleopathology, 1(1): 24-34.

Morales, Daniel (1998). Historia arqueológica del Perú. Compendio Histórico del Perú. T. I. Milla Batres. Lima.

Ortner, D. J. (2003). Identification of Pathological Conditions in Human Skeletal Remains. San Diego: Academic Press.

Poma de Ayala, Guamán (1962 [1615]). Nueva corónica y buen gobierno. Caracas: Biblioteca Ayacucho.

Reimer, P. J., E. Bard, A. Bayliss, J. W. Beck, P. G. Blackwell, C. Bronk Ramsey, P. M. Grootes, T. P. Guilderson, H. Haflidason, I. Hajdas, C. Hatte, T. J. Heaton, D. L. Hoffmann, A. G. Hogg, K. A. Hughen, K. F. Kaiser, B. Kromer, S. W. Manning, M. Niu, R. W. Reimer, D. A. Richards, E. M. Scott, J. R. Southon, R. A. StafF, C.s.m. Turney, $\&$ J. Van Der Plicht (2013). IntCal13 and Marine13 Radiocarbon Age Calibration Curves 0-50,000 Years cal BP. Radiocarbon,55(4): 1869-1887.

Reimers E., L. Pena; R. Sarmiento-Herrera, B. Pinero \& Y. M. Rosa (2008). Exostosis auriculares: una lesión del presente y del pasado. Revista espanyola deenfermedades metabólicasóseas, 17: 112-3.

Rostworowsкi, María (2005). Recursos naturales renovables y pesca, siglos XVI y XVII. Instituto de Estudios Peruanos, Lima.

Rowe, Ann (2014). Technical Reflections of HighlandCoastal Relationships in Late Pre-Hispanic Tunics from Chillon and Chancay, in Denise Arnold with Penelope Dransart (eds.) Textiles, Technical Practice and Power in the Andes: 159-191. Archetype Publications, London.

Shimada, Izumi; Klaus H. D; Segura R.; Matsumoto G. (2015). Living with the dead. Conception and treatment of the dead on the Peruvian Coast, in I. Shimada, J. L. Fitzimmons (eds.) Living with the dead in the Central Andes: $101-172$. The University of the Arizona Press. 
Tello, Julio C. (2009). Paracas Wari Kayan. Cuadernos de Investigación del Archivo Tello. 9. Museo de Arqueología y Antropología de la Universidad Nacional Mayor de San Marcos. Lima.

VAn Dalen, Pieter (2008). Los ecosistemas arqueológicos en la cuenca baja del río Chancay-Huaral. Su importancia para el desarrollo de las formaciones sociales prehispánicas. Lima.

Van Dalen, Pieter (2011). Investigaciones en el complejo arqueológico de Lumbra, un asentamiento tardío del valle medio del río Chancay-Huaral. Investigaciones sociales 28: 285- 302. Instituto de Investigaciones Sociales, Facultad de Ciencias Sociales. Universidad Nacional Mayor de San Marcos. Lima.

Van Dalen, Pieter (2013). Contextos funerarios Chancay en Macatón, Valle de Chancay-Huaral. Arqueología y Sociedad 25: 259-302. Museo de Arqueología y Antropología, Universidad Nacional Mayor de San Marcos. Lima.

Van Dalen, Pieter (2016a). Estrategias de dominación Tawantinsuyu en el complejo arqueológico de Lumbra, valle medio del río Chancay, provincia de Huaral. Universidad Nacional Mayor de San Marcos. Lima.

Van Dalen, Pieter (2016b). El hallazgo de cuerpos tatuados en Cerro Colorado, valle de Huaura, Costa norcentral peruana, in Cuaderno de Resumenes, VII Congresso Internacional Imagens da Morte. Tempos e Espacos da Morte na Sociedade: 102- 103.Sao Paolo, Brasil.

Van Dalen, Pieter (2017). Sacachispa: un cementerio de agricultores de la cultura Chancay en Huando, Huaral. Lima.

Van Dalen, Pieter; Tello, Roberto y Grados, Hans (2016). Un contexto funerario del Horizonte Medio procedente de Cerro Colorado, Huacho. Arqueología y Sociedad 30: 407-424. Museo de Arqueología y Antropología, Universidad Nacional Mayor de San Marcos. Lima.

Walker P. L.; Bathurst R. R.; Richman R.; Gjerdrum T.; Andrushro V. A. (2009). The Causes of Porotic Hyperostosis and Cribra Orbitalia: A Reappraisal of the Iron-Deficiency-Anemia Hypothesis. American Journal of Physical Anthropology, 39:109-125. 